Article

\title{
An Optimization of the Signal-to-Noise Ratio Distribution of an Indoor Visible Light Communication System Based on the Conventional Layout Model
}

\author{
Xiangyang Zhang ${ }^{1} \oplus$, Nan Zhao ${ }^{1}$, Fadi Al-Turjman ${ }^{2} \oplus$, Muhammad Bilal Khan ${ }^{1}$ \\ and Xiaodong Yang ${ }^{1, *}$ \\ 1 School of Electronic Engineering, Xidian University, Xi'an 710071, China; \\ xyzhang_5@stu.xidian.edu.cn (X.Z.); nzhao_3@stu.xidian.edu.cn (N.Z.); bilal@stu.xidian.edu.cn (M.B.K.) \\ 2 Artificial Intelligence Engineering Department, Research Centre for AI and IoT, Near East University, \\ 99138 Nicosia, Mersin 10, Turkey; fadi.alturjman@neu.edu.tr \\ * Correspondence: xdyang@xidian.edu.cn
}

Received: 12 September 2020; Accepted: 23 October 2020; Published: 29 October 2020

check for updates

\begin{abstract}
For an actual visible light communication system, it is necessary to consider the uniformity of indoor illumination. Most of the existing optimization schemes, however, do not consider the effect of the first reflected light, and do not conform to the practical application conventions, which increases the actual cost and the complexity of system construction. In this paper, considering the first reflected light and based on the conventional layout model and the classic indoor visible light communication model, a scheme using the parameter $Q$ to determine the optimal layout of channel quality is proposed. We determined the layout, and then carried out a simulation. For comparison, the normal layout and the optimal layout of illumination were also simulated. The simulation results show that the illuminance distributions of the three layouts meet the standards of the International Organization for Standardization. The optimal layout of channel quality in the signal-to-noise ratio distribution, maximum delay spread distribution, and impulse response is obviously better than the optimal layout of illumination. In particular, the effective area percentage of the optimal layout of channel quality is increased by $0.32 \%$ and $6.08 \%$ to $88.80 \%$ as compared with the normal layout's $88.48 \%$ and the optimal layout of illumination's $82.72 \%$. However, compared with the normal layout, the advantages are not very prominent.
\end{abstract}

Keywords: impulse response; inter-symbol interference; light-emitting diode; maximum delay spread; signal-to-noise ratio; visible light communication

\section{Introduction}

For an actual visible light communication (VLC) system, it is necessary to consider the uniformity of indoor illumination, so it is preferred to use light-emitting diodes (LEDs) with a larger half-power angle, which enhances the multipath effect while causing inter-symbol interference (ISI) and reducing the signal-to-noise ratio (SNR). In order to improve the performance of a VLC system, various optimization schemes have been proposed [1-5].

An optimization scheme based on an evolutionary algorithm is proposed to modify the optical intensity of LED transmitters for reducing the signal power fluctuation extent [1]. A new arrangement scheme of LED lamps is proposed, and the SNR fluctuation is reduced from 14.5 to $0.9 \mathrm{~dB}$ [2]. A hybrid scheme that combines spotlighting with uniform lighting is proposed to achieve uniform illumination and high data rate [3]. An optimization scheme based on light-shaping diffusers is proposed to achieve 
uniform power distribution [4]. An optimization scheme based on the Lambertian order is proposed to improve the performance of a VLC system [5].

According to published papers [6,7], it is necessary to consider the effect of the first reflected light, but most of the existing optimization schemes only consider the direct radiation, and the optimization results usually do not conform to the practical application conventions, which increase the actual cost and the complexity of system construction. In this paper, considering the first reflected light and based on the conventional layout model and the classic indoor VLC model, the normal layout, the optimal layout of illumination, and the optimal layout are simulated, and the relevant data are analyzed.

Following the introduction, this paper is organized in four sections. In Section 2, the basic theories and calculation formulas of VLC simulation are shown. In Section 3, the data and indoor environment used in the simulation are shown, the parameter $Q$ is defined, and the three layouts are determined. In Section 4, the illuminance distribution, SNR distribution, maximum delay spread (M-DS) distribution, and impulse response (IR) of the three layouts are shown and analyzed. Finally, our conclusions are given in Section 5.

\section{Basic Theories of VLC}

This section is divided into three parts, which respectively explain the basic theories and calculation formulas used in the simulation of illuminance, optical radiation power, and channel quality in VLC. In existing papers $[5,6]$, it is assumed that the light source is a near-Lambertian light source and the reflective surface is a standard Lambertian surface. These two assumptions are used in this paper. Some basic photometric parameters are described below.

The basic physical quantity used in optical radiometry is the radiant flux or radiant power, symbolized by $\Phi_{\mathrm{e}}$ in watts (W). Photometry is a discipline that studies the senses of human eyes. The units used are based on a physical basis and the characteristics of the human eye. Therefore, all units in photometry are artificial.

Luminous flux indicates the visual intensity of the human eye caused by radiant flux, which is the physical quantity derived from the radiant flux $\Phi_{\mathrm{e}}$, that is, the photometric quantity of the radiant flux, based on the effect of the radiation on the standard photometric observer of the International Commission on illumination (CIE). Luminous flux is an intrinsic property of a light source. The symbol is $\Phi_{\mathrm{v}}$ and the unit is lumen $(\mathrm{lm}=\mathrm{cd} \cdot \mathrm{sr})$ :

$$
\Phi_{\mathrm{v}}=K_{\mathrm{m}} \int_{360}^{830} \Phi_{\mathrm{e}}(\lambda) V(\lambda) d \lambda
$$

where $V(\lambda)$ is the photopic optical efficiency function, as shown in Figure $1 ; K_{\mathrm{m}}$ is the photopic optical efficiency at $\lambda=555 \mathrm{~nm}$, and is also the maximum of $V(\lambda)$, one of the photometric constants, $K_{\mathrm{m}}=683 \mathrm{~lm} / \mathrm{W}$.

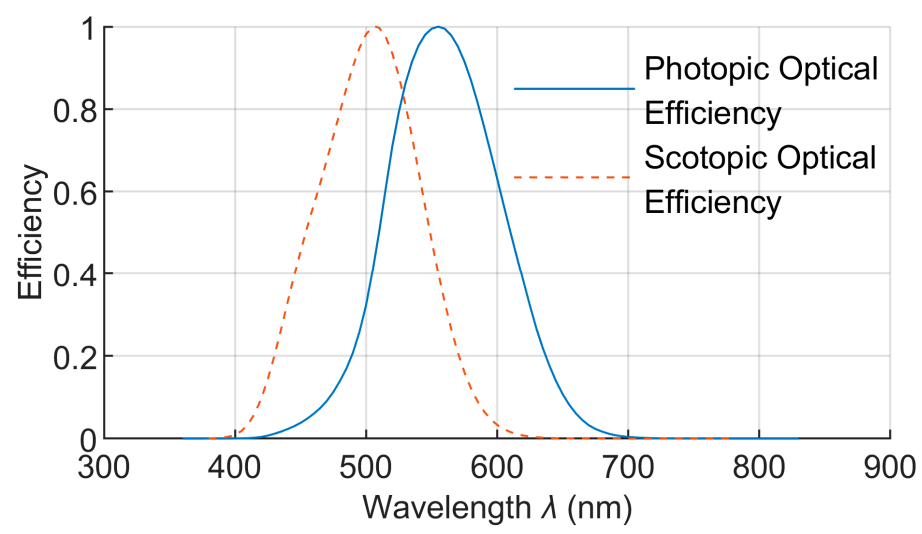

Figure 1. Optical efficiency function. 
Luminous intensity indicates the illuminating characteristic of a light source in a given direction, which is defined as the magnitude of the luminous flux $\Phi_{\mathrm{v}}$ per unit solid angle $\Omega$ in a given direction. The symbol is $I_{\mathrm{v}}$ and the unit is candela (cd):

$$
I_{\mathrm{v}}=\frac{d \Phi_{\mathrm{v}}}{d \Omega}
$$

Luminance indicates the illuminating characteristic of the illuminating surface in a given position and direction, which is defined as the illuminating intensity $I_{\mathrm{v}}$ per unit cross-section perpendicular to the direction. The symbol is $L_{\mathrm{V}}$ and the unit is candela per square meter $\left(\mathrm{cd} / \mathrm{m}^{2}\right)$ :

$$
L_{\mathrm{v}}=\frac{I_{\mathrm{V}}}{d \mathrm{~A} \cdot \cos \theta}=\frac{d \Phi_{\mathrm{v}}}{d \mathrm{~A} \cdot \cos \theta \cdot d \Omega}
$$

where $d \mathrm{~A}$ is the surface element of the beam section; $\theta$ is the angle between the normal of the surface element and the given direction, as shown in Figure 2.

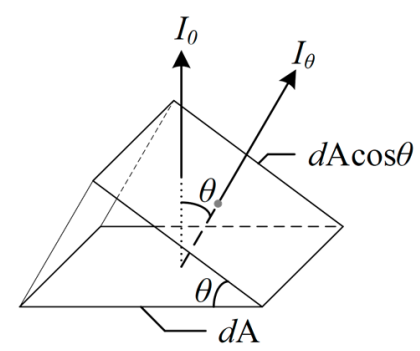

Figure 2. Luminance definition.

Illuminance indicates the light-receiving characteristic of the illuminated surface in a given position, which is defined as the luminous flux $\Phi_{\mathrm{v}}$ received on the unit tangent plane on the fixed point. The symbol is $E_{\mathrm{v}}$ and the unit is $\operatorname{lux}\left(\mathrm{lx}=\mathrm{cd} \cdot \mathrm{sr} / \mathrm{m}^{2}\right)$ :

$$
E_{\mathrm{v}}=\frac{d \Phi_{\mathrm{v}}}{d \mathrm{~A}}=\frac{I_{\mathrm{v}} \cdot d \Omega}{d \mathrm{~A}}=\frac{I_{\mathrm{v}}}{d \mathrm{~A}} \cdot d \Omega=\frac{I_{\mathrm{v}}}{d \mathrm{~A}} \cdot \frac{d \mathrm{~A} \cdot \cos \theta}{r^{2}}=\frac{I_{\mathrm{v}} \cdot \cos \theta}{r^{2}}
$$

where $d \mathrm{~A}$ is the surface element of the tangent plane on the fixed point; $\theta$ is the angle between the normal of the surface element and the direction from the fixed point to the point source; $r$ is the distance between the fixed point and the point source, as shown in Figure 3.

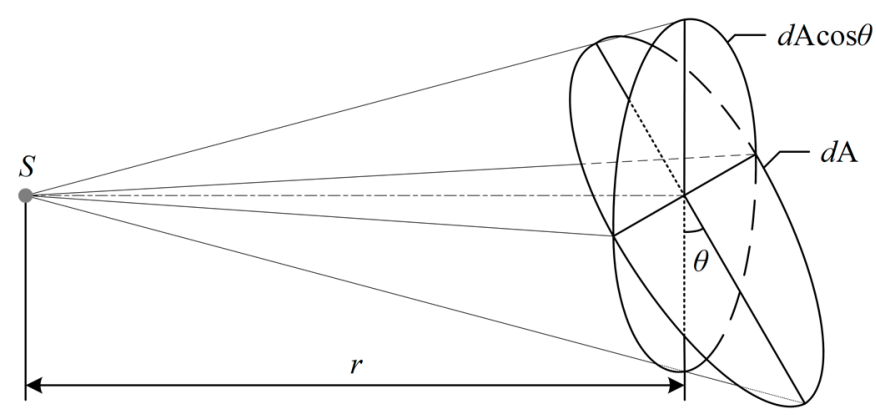

Figure 3. Illuminance definition.

The luminous intensity of a standard Lambertian source (standard Lambertian surface) conforms to the law of cosine, as shown in Figure 4. 


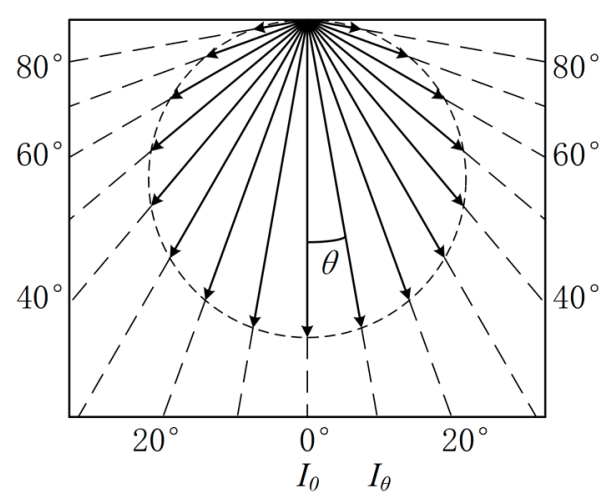

Figure 4. Standard Lambertian source luminous intensity diagram.

The luminous intensity $I_{\mathrm{v}, \theta}$ at a certain angle is equal to the light intensity $I_{\mathrm{v}, 0}$ in the axial direction of the light source multiplied by the cosine of the direction angle:

$$
I_{\mathrm{v}, \theta}=I_{\mathrm{v}, 0} \cdot \cos \theta
$$

From Equations (3) and (5), the luminance of a standard Lambertian source (standard Lambertian surface) is independent of the direction, as shown in Figure 5:

$$
L_{\mathrm{v}, \theta}=\frac{I_{\mathrm{v}, \theta}}{d \mathrm{~A} \cdot \cos \theta}=\frac{I_{\mathrm{v}, 0} \cdot \cos \theta}{d \mathrm{~A} \cdot \cos \theta}=\frac{I_{\mathrm{v}, 0}}{d \mathrm{~A}}
$$

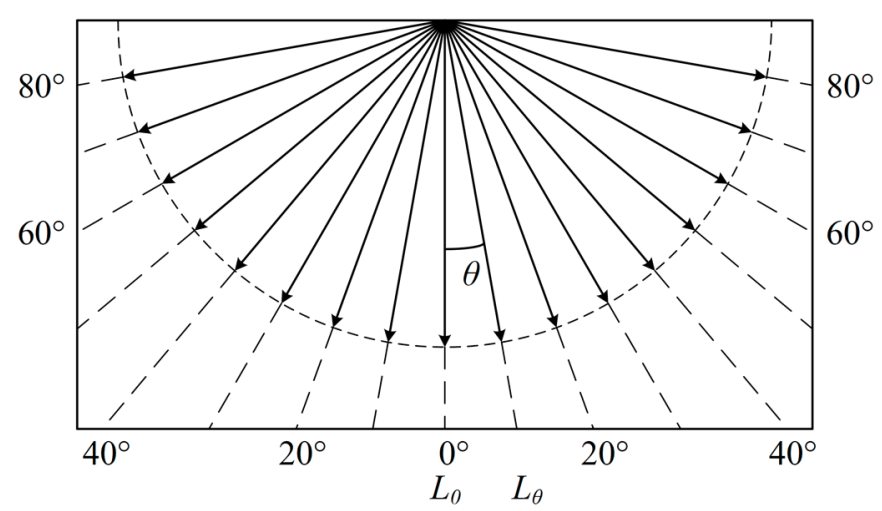

Figure 5. Standard Lambertian source luminance diagram.

Symbolizing the luminance of a standard Lambertian source as $L_{\mathrm{V}}$ and the area as $d \mathrm{~A}$, the total luminous flux $\Phi_{\mathrm{v}}$ radiated in the entire hemispherical surface, SP, is given in Equation (7):

$$
\Phi_{\mathrm{V}}=\int_{\mathrm{SP}} I_{\mathrm{V}} d \Omega=\int_{\mathrm{SP}} L_{\mathrm{V}} d \mathrm{~A} \cos \theta d \Omega=2 \pi L_{\mathrm{V}} d \mathrm{~A} \int_{0}^{\frac{\pi}{2}} \cos \theta \sin \theta d \theta=\pi L_{\mathrm{V}} d \mathrm{~A}
$$

Therefore, the luminous exitance $M_{\mathrm{V}}$ and the luminance $L_{\mathrm{V}}$ of a standard Lambertian source are in the following relationship:

$$
M_{\mathrm{v}}=\frac{\Phi_{\mathrm{v}}}{d \mathrm{~A}}=\frac{\pi L_{\mathrm{v}} d \mathrm{~A}}{d \mathrm{~A}}=\pi L_{\mathrm{v}}
$$

In the LED industry, people use the half power angle $\theta_{1 / 2}$ as a measure of the angle of illumination. The half power angle $\theta_{1 / 2}$ is defined as the angle between the axial direction and the direction in which the luminous intensity is half with the axial direction. From Equation (5), the half power angle $\theta_{1 / 2}$ of the standard Lambertian source is $60^{\circ}$. 
In practical applications, the LED half power angle $\theta_{1 / 2}$ is often not equal to $60^{\circ}$. Such an LED is called a near-Lambertian light source, and the luminous intensity function is expressed as:

$$
I_{\theta}=I_{0} \cos ^{m} \theta
$$

where $m$ is the order of the Lambertian source. Combined with the definition of the half power angle $\theta_{1 / 2}$, the following is obtained:

$$
m=-\frac{\ln 2}{\ln \left(\cos \theta_{1 / 2}\right)}
$$

So, we know that the order of the Lambertian source $m$ and the half power angle $\theta_{1 / 2}$ correspond to each other. The order of the Lambertian source $m$ can be obtained by the half power angle $\theta_{1 / 2}$, and the normalized luminous intensity distribution function curve is plotted, as shown in Figure 6.

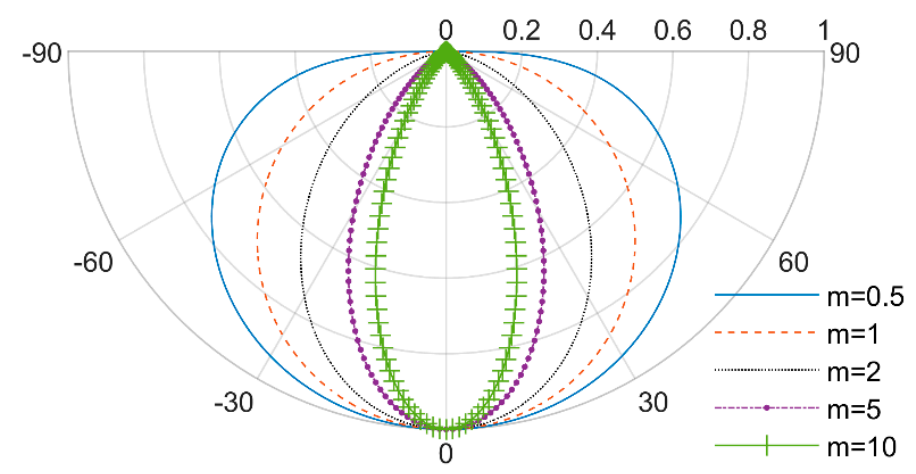

Figure 6. Normalized near-Lambertian source luminous intensity diagram.

From Equations (3) and (9), the luminance of a near-Lambertian source is related to direction, and the normalized luminance distribution is shown in Figure 7:

$$
L_{\mathrm{v}, \theta}=\frac{I_{\mathrm{v}, \theta}}{d \mathrm{~A} \cdot \cos \theta}=\frac{I_{\mathrm{v}, 0} \cos ^{m} \theta}{d \mathrm{~A} \cdot \cos \theta}=\frac{I_{\mathrm{v}, 0}}{d \mathrm{~A}} \cos ^{m-1} \theta
$$

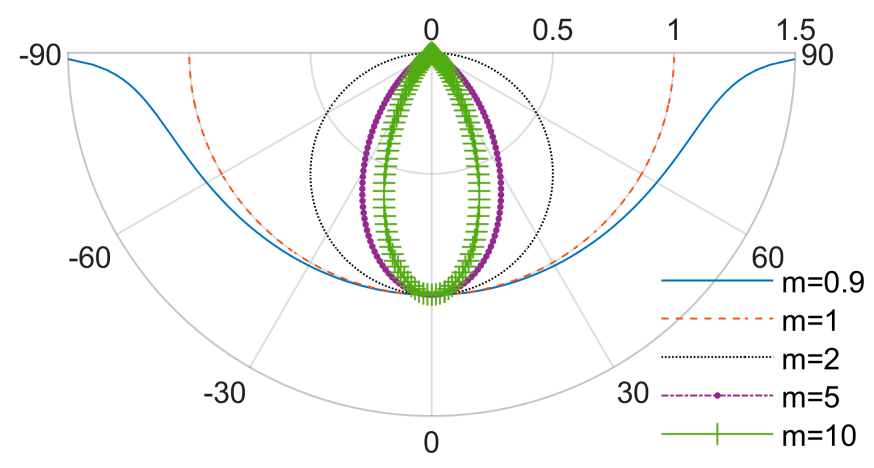

Figure 7. Normalized near-Lambertian source luminance diagram.

Figure 8 is a diagram of indoor VLC communication. We know that the indoor VLC communication links are divided into two categories: line of sight (LOS) and non-line of sight (NLOS). It has been proved that the proportion of the secondary reflected light to the total is too small [6]. In this paper, only direct light and first reflected light are considered. 


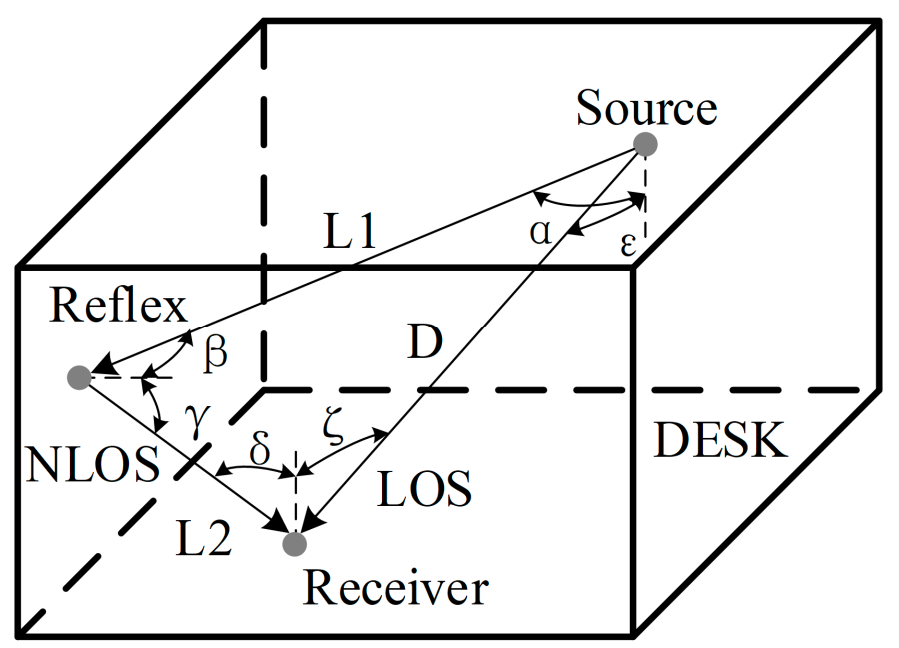

Figure 8. Indoor visible light communication (VLC) communication diagram.

\subsection{Horizontal Illuminance of LOS and NLOS}

\subsubsection{Horizontal Illuminance of LOS}

From Equations (4) and (9), the horizontal illuminance of LOS, $E_{\mathrm{v}, \mathrm{LOS}}$, is known:

$$
E_{\mathrm{V}, \mathrm{LOS}}=\frac{I_{0} \cos ^{m} \theta \cos \zeta}{D^{2}}
$$

where $I_{0}$ is the axial luminous intensity of LED lamps.

\subsubsection{Horizontal Illuminance of NLOS}

From Equation (12), the illuminance at the reflection point on the wall of NLOS, $E_{\mathrm{v}, \mathrm{NLOS} \text {,Ref, }}$ is known:

$$
E_{\mathrm{V}, \mathrm{NLOS}, \mathrm{Ref}}=\frac{I_{0} \cos ^{m} \alpha \cos \beta}{L_{1}^{2}}
$$

From Equation (13), the luminous emittance at the reflection point on the wall of NLOS, $M_{\mathrm{V}, \mathrm{NLOS} \text {, Ref }}$ is known:

$$
M_{\mathrm{v}, \mathrm{NLOS}, \mathrm{Ref}}=\rho E_{\mathrm{v}, \mathrm{NLOS}, \mathrm{Ref}}=\frac{\rho I_{0} \cos ^{m} \alpha \cos \beta}{L_{1}^{2}}
$$

where $\rho$ is the reflectivity of the wall.

From Equations (3), (8) and (14), the luminous intensity in the normal direction at the reflection point of NLOS, $I_{\mathrm{V}, \mathrm{NLOS}, \text { Ref }}$, is known:

$$
I_{\mathrm{V}, \mathrm{NLOS}, \text { Ref }}=L_{\mathrm{v}, \mathrm{NLOS}, \operatorname{Ref}} d A_{\mathrm{WALL}}=\frac{M_{\mathrm{v}, \mathrm{NLOS}, \text { Ref }} d A_{\mathrm{WALL}}}{\pi}=\frac{\rho I_{0} \cos ^{m} \alpha \cos \beta d A_{\mathrm{WALL}}}{\pi L_{1}^{2}}
$$

where $d A_{\text {WALL }}$ is the reflective area of a small region.

From Equations (4), (5) and (15), the horizontal illuminance of NLOS, $E_{\mathrm{V}, N L O S}$, is known:

$$
E_{\mathrm{V}, \mathrm{NLOS}}=\frac{I_{\mathrm{v}, \mathrm{NLOS}, \mathrm{Ref}} \cos \gamma \cos \delta}{L_{2}^{2}}=\frac{\rho I_{0} \cos ^{m} \alpha \cos \beta \cos \gamma \cos \delta d A_{\mathrm{WALL}}}{\pi L_{1}^{2} L_{2}^{2}}
$$




\subsection{Received Power of LOS and NLOS}

Analogously to the definition of luminous intensity, in radiometry, the radiation intensity is defined as the radiant flux $\Phi_{\mathrm{e}}$ per unit solid angle $\Omega$ in a given direction. The symbol is $I_{\mathrm{e}}$ and the unit is watts per solid angle $(\mathrm{W} / \mathrm{sr})$ :

$$
I_{\mathrm{e}}=\frac{d \Phi_{\mathrm{e}}}{d \Omega}
$$

Because luminous intensity and radiation intensity are descriptions of the same physical phenomenon from different aspects, according to Equation (9), the radiation intensity of a near-Lambertian source can be assumed as follows:

$$
I_{\mathrm{e}}=I_{\mathrm{e}, 0} \cos ^{m} \theta=V_{m} \Phi_{\mathrm{e}} \cos ^{m} \theta
$$

where $V_{m}$ is a variable associated with $m$ and determining the relationship between $\Phi_{\mathrm{e}}$ and $I_{\mathrm{e}}$.

For a single-sided LED, the total radiant flux $\Phi_{\mathrm{e}}$ radiated throughout the hemispherical surface SP can be calculated as:

$$
\Phi_{\mathrm{e}}=\int_{\mathrm{SP}} I_{\mathrm{e}}(\theta) d \Omega=2 \pi \int_{0}^{\frac{\pi}{2}} I_{\mathrm{e}}(\theta) \sin \theta d \theta=2 \pi \Phi_{\mathrm{e}} V_{m} \int_{0}^{\frac{\pi}{2}} \cos ^{m} \theta \sin \theta d \theta
$$

From Equations (18) and (19), the radiant intensity $I_{\mathrm{e}}$ is known:

$$
I_{\mathrm{e}}=\frac{(m+1) \Phi_{\mathrm{e}} \cos ^{m} \theta}{2 \pi}
$$

\subsubsection{Received Power of LOS}

From Equations (1), (12) and (20), the received power of LOS, PLOS, is known [6]:

$$
P_{\mathrm{LOS}}= \begin{cases}\frac{(m+1) A_{\mathrm{R}}}{2 \pi D^{2}} P_{\mathrm{S}} \cos ^{m} \theta \cos \zeta g(\zeta) T_{\mathrm{S}}(\zeta) & 0 \leq \zeta \leq \Psi_{\mathrm{C}} \\ 0 & \zeta \leq \Psi_{\mathrm{C}}\end{cases}
$$

where $A_{\mathrm{R}}$ is the physical area of the detector in a photo diode (PD); $P_{\mathrm{S}}$ is the transmitted optical power of the source; $T_{S}(\zeta)$ is the gain of an optical filter; $\Psi_{\mathrm{c}}$ is the width of the field of vision (FOV) at a receiver; $g(\zeta)$ is the gain of an optical concentrator.

$g(\zeta)$ is known [6], and is given in Equation (22):

$$
g(\zeta)= \begin{cases}\frac{n^{2}}{\sin ^{2} \Psi_{c}} & 0 \leq \zeta \leq \Psi_{c} \\ 0 & \zeta \leq \Psi_{c}\end{cases}
$$

where $n$ is the refractive index.

\subsubsection{Received Power of NLOS}

From Equations (1), (16) and (20), the received power of NLOS, $P_{\mathrm{NLOS}}$, is known [6]:

$$
P_{\mathrm{NLOS}}= \begin{cases}\frac{(m+1) A_{\mathrm{R}}}{2 \pi L_{1}^{2} L_{2}^{2}} P_{\mathrm{S}} \rho \cos ^{m} \alpha \cos \beta \cos \gamma \cos \delta d A_{\mathrm{WALL}} g(\delta) T_{\mathrm{S}}(\delta) & 0 \leq \delta \leq \Psi_{\mathrm{c}} \\ 0 & \delta \leq \Psi_{\mathrm{c}}\end{cases}
$$

\subsection{Channel Quality}

Channel noise is divided into three parts: ISI noise, shot noise, and thermal noise. 
In the indoor VLC communication system, the signal-to-noise ratio at the receiving end is expressed as follows [6]:

$$
S N R=\frac{S}{N}=\frac{\gamma^{2} P_{r S i g n a l}^{2}}{\sigma_{\text {shot }}^{2}+\sigma_{\text {thermal }}^{2}+\gamma^{2} P_{r I S I}^{2}}
$$

where $\gamma$ is the detector responsivity; $P_{r \text { Signal }}$ is the signal power; $\sigma^{2}$ shot is the shot noise; $\sigma^{2}$ thermal is the thermal noise; $P_{r I S I}$ is the ISI noise power.

\subsubsection{ISI Noise}

ISI noise is caused by ISI power. In a VLC system, the power received by the PD is divided into signal power and ISI power. When a signal is transmitted, during the first arriving signal operation, the power of all the paths is the signal power. After the first arriving signal is completed, the power of all the paths is the ISI power, as shown in Figure 9.

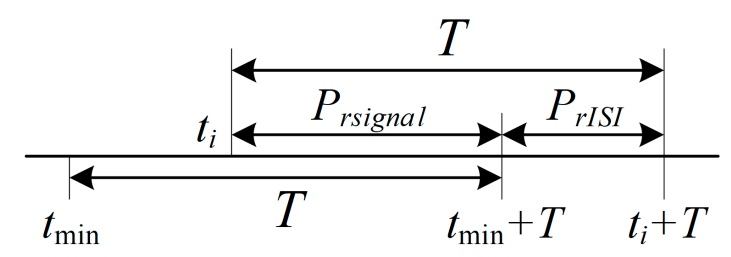

Figure 9. Signal power and inter-symbol interference (ISI) power diagram.

In Figure $9, t_{\min }$ is the earliest arrival time of the signals in all paths, $t_{i}$ is the arrival time of the signal in the path, and $T$ is the symbol period.

\subsubsection{Shot Noise}

Shot noise originates from active components in electronic devices and is caused by uneven electron emission. In this system, it results from the photocurrent caused by LED illumination and the dark current caused by ambient illumination.

The shot noise caused by LED illumination, $\sigma^{2}$ shot,LED, is known [6]:

$$
\sigma_{\text {shot }, \text { LED }}^{2}=2 q \gamma\left(P_{r \text { Signal }}+P_{r I S I}\right) B
$$

where $q$ is the electronic charge; $B$ is equivalent noise bandwidth, and it is equal to the data rate.

The shot noise caused by ambient illumination, $\sigma^{2}$ shot,bg, is known [6]:

$$
\sigma_{\text {shot }, b g}^{2}=2 q I_{b g} I_{2} B
$$

where $I_{b g}$ is background current; $I_{2}$ is one of the noise band width factors.

\subsubsection{Thermal Noise}

Thermal noise is also called resistance noise, originates from passive components in electronic devices, and is caused by electronic Brownian motion. In this system, it results from feedback resistors and field effect transistor (FETs).

The thermal noise resulting from feedback resistors, $\sigma^{2}$ thermal,Res, is known [6]:

$$
\sigma_{\text {thermal, Res }}^{2}=\frac{8 \pi k T_{k}}{G} \eta A_{\mathrm{R}} I_{2} B^{2}
$$

where $k$ is Boltzmann's constant; $T_{k}$ is the absolute temperature; $\eta$ is the fixed capacitance of the photo detector per unit area; $G$ is the open-loop voltage gain; $A_{R}$ is the physical area of the detector in a PD; $B$ is equivalent noise bandwidth, and it is equal to the data rate. 
The thermal noise resulting from FETs, $\sigma^{2}$ thermal,FET, is known [6]:

$$
\sigma_{\text {thermal }, F E T}^{2}=\frac{16 \pi^{2} k T_{k} \Gamma}{g_{m}} \eta^{2} A_{\mathrm{R}}^{2} I_{3} B^{3}
$$

where $\Gamma$ is the FET channel noise factor; $I_{3}$ is one of the noise band width factors; $g_{m}$ is the FET transconductance; $\eta$ is the fixed capacitance of the photo detector per unit area; $A_{\mathrm{R}}$ is the physical area of the detector in a PD; $B$ is the equivalent noise bandwidth, and it is equal to the data rate.

\section{Simulation Data and LED Layouts}

This section is divided into two parts. The first part describes the data used in the simulation. The second part determines the three different LED layouts (normal, optimal illumination, optimal channel quality). All the simulations in this paper were completed using MATLAB software.

\subsection{Simulation Data}

\subsubsection{Conventional LED Layout Model}

The conventional indoor LEDs layout model is shown in Figure 10. The room size is $5 \mathrm{~m} \times 5 \mathrm{~m}$, the height of the table is $0.75 \mathrm{~m}$ from the floor, and the height of the lamps' light is $2.5 \mathrm{~m}$ from the floor. The four lamps are symmetrically distributed in the room, and each lamp consists of $60 \times 60$ LED chips with a pitch of $0.01 \mathrm{~m} \mathrm{[6].}$

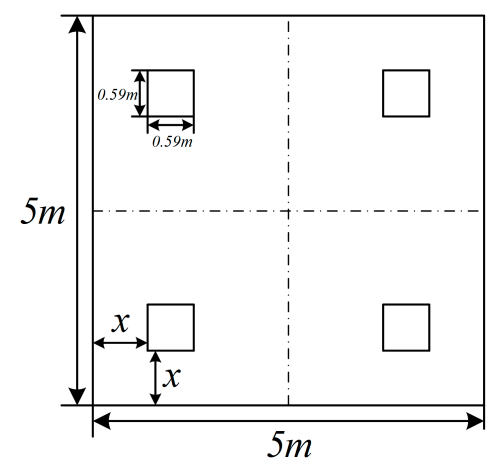

Figure 10. Light-emitting diode (LED) layout model diagram.

From Figure 10, it is known that if the distance $x$ between the edge of one of the lamps and the edge of the adjacent wall is determined, the overall layout of the indoor LEDs is uniquely determined.

\subsubsection{Simulation Parameter}

The simulation settings are shown in Table 1.

Table 1. Simulation calculation settings.

\begin{tabular}{cc}
\hline Item & Data \\
\hline Reflectivity of the wall, $\rho$ & 0.7 \\
Reflective area of small region, $d A_{\text {WALL }}$ & $5 \times 5 \mathrm{~cm}$ \\
Simulation space accuracy & $1 \mathrm{~cm}$ \\
Data rate & $100 \mathrm{Mb} / \mathrm{s}$ \\
Modulation & On-Off Keying (OOK) \\
\hline
\end{tabular}

The parameters of the LED chips are shown in Table 2. 
Table 2. Parameters of the LED chips.

\begin{tabular}{cc}
\hline Item & Data \\
\hline Center luminous intensity, $I_{\mathrm{v}, 0}$ & $0.73 \mathrm{~cd}$ \\
Semi-angle at half power, $\theta_{1 / 2}$ & $70^{\circ}$ \\
Transmitted optical power, $P_{\mathrm{S}}$ & $20 \mathrm{~mW}$ \\
\hline
\end{tabular}

The parameters of the PDs are shown in Table 3.

Table 3. Parameters of the photo diodes (PDs).

\begin{tabular}{cc}
\hline Item & Data \\
\hline Detector physical area, $A_{\mathrm{R}}$ & $1 \mathrm{~cm}^{2}$ \\
Gain of an optical filter, $T_{\mathrm{S}}$ & 1 \\
FOV, $\Psi_{\mathrm{c}}$ & $60^{\circ}$ \\
Refractive index of a lens, $n$ & 1.5 \\
O/E conversion efficiency, $\gamma$ & $0.53 \mathrm{~A} / \mathrm{W}$ \\
\hline
\end{tabular}

The parameters of the noise calculation are shown in Table 4.

Table 4. Parameters of the noise calculation.

\begin{tabular}{cc}
\hline Item & Data \\
\hline Equivalent noise bandwidth, $B$ & $100 \mathrm{Mb} / \mathrm{s}$ \\
Background current, $I_{b g}$ & $5100 \mu \mathrm{A}$ \\
Noise bandwidth factor, $I_{2}$ & 0.562 \\
Noise bandwidth factor, $I_{3}$ & 0.0868 \\
Absolute temperature, $T_{k}$ & $298 \mathrm{~K}$ \\
Fixed capacitance, $\eta$ & $112 \mathrm{pF} / \mathrm{cm}^{2}$ \\
Open-loop voltage gain, $G$ & 10 \\
FET channel noise factor, $\Gamma$ & 1.5 \\
FET transconductance, $g_{m}$ & $30 \mathrm{mS}$ \\
\hline
\end{tabular}

\subsection{LED Layout}

\subsubsection{Normal Layout}

The normal layout is the equally spaced layout used in [6]. Each lamp is located at the center of the respective area, and $x$ is $0.955 \mathrm{~m}$, as shown in Figure 11.

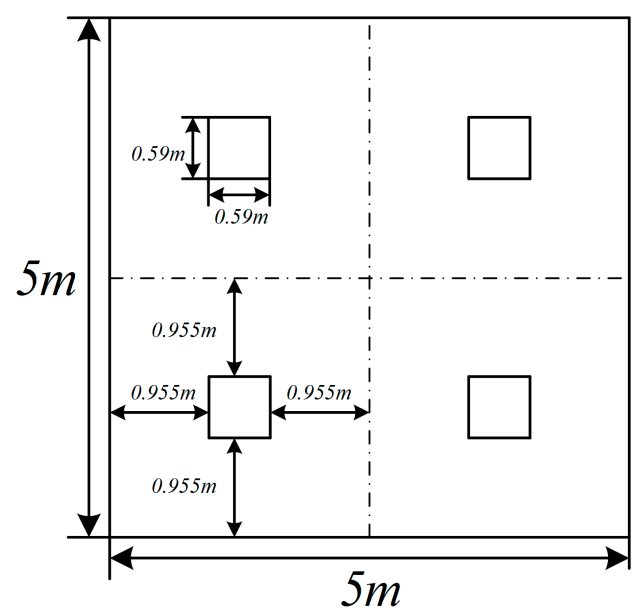

Figure 11. Normal layout diagram. 


\subsubsection{Optimal Layout of Illumination}

Drawing on existing literature [2], we use the parameter $Q_{E}$ to evaluate the horizontal illuminance.

$$
Q_{E}=\frac{\overline{E_{\mathrm{v}}}}{\sqrt{\operatorname{var}\left(E_{\mathrm{v}}\right)}}
$$

where $\overline{E_{\mathrm{v}}}$ is the mean of horizontal illuminance; $\operatorname{var}\left(E_{\mathrm{v}}\right)$ is the variance of horizontal illuminance. The larger $Q_{E}$ is, the more uniform the horizontal illumination distribution will be.

The relationship between $x$ and $Q_{E}$ is shown in Figure 12. As can be seen from the figure, when $x$ is $0.77 \mathrm{~m}$, $Q_{E}$ reaches the maximum value of 8.0945 , meaning that this LED layout is the optimal layout of illumination under the conventional indoor LED layout model.

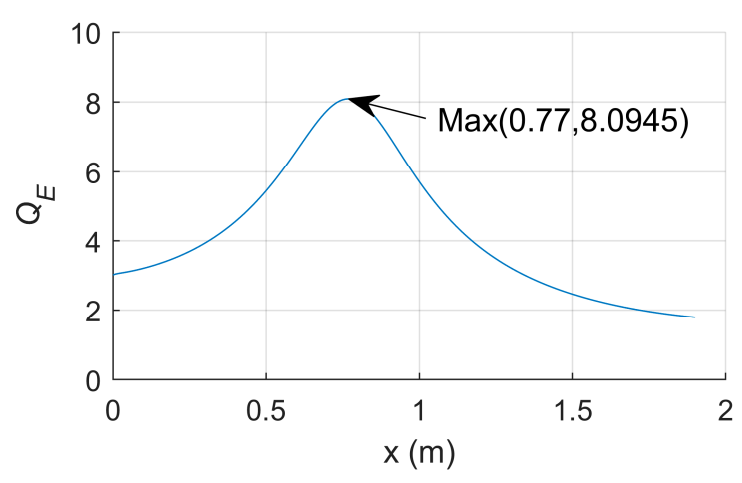

Figure 12. Relationship between $x$ and $Q_{E}$.

\subsubsection{Optimal Layout of Channel Quality}

Drawing on existing literature [4], we use the parameter $Q_{S N R}$ to evaluate the channel quality.

$$
Q_{S N R}=\frac{\overline{S N R}}{\sqrt{\operatorname{var}(S N R)}}
$$

where $\overline{S N R}$ is the mean of the SNR; $\operatorname{var}(S N R)$ is the variance of the SNR. The larger $Q_{S N R}$ is, the more uniform the SNR distribution will be.

The relationship between $x$ and $Q_{S N R}$ is shown in Figure 13. As can be seen from the figure, when $x$ is $0.97 \mathrm{~m}, Q_{S N R}$ reaches the maximum value of 18.1616 , meaning that this LED layout is the optimal layout of channel quality under the conventional indoor LED layout model.

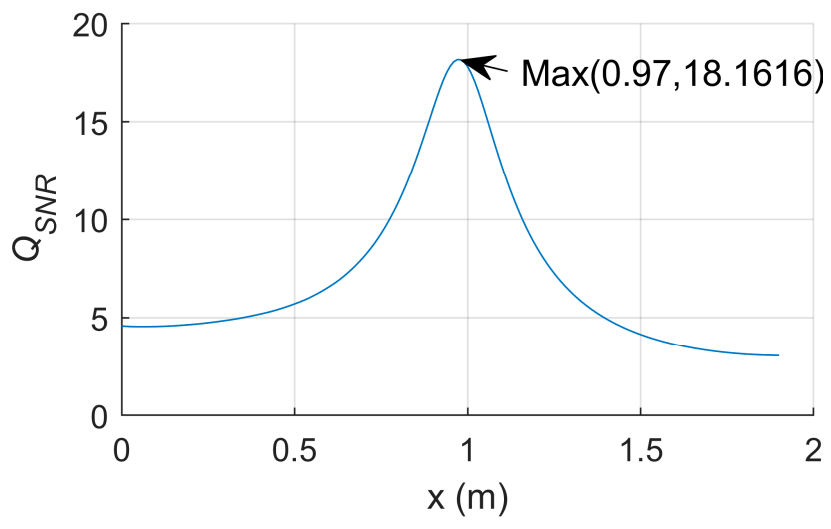

Figure 13. Relationship between $x$ and $Q_{S N R}$. 


\section{Data Analysis}

In Section 3, three kinds of LED layouts were determined. In this section, we compare and analyze the illuminance distribution, SNR distribution, M-DS distribution, and IR of the three layouts.

\subsection{Illuminance Distribution}

The illuminance distribution of the three different LED layouts is simulated. The simulation results are shown in Figure 14, and the data comparison is shown in Table 5.

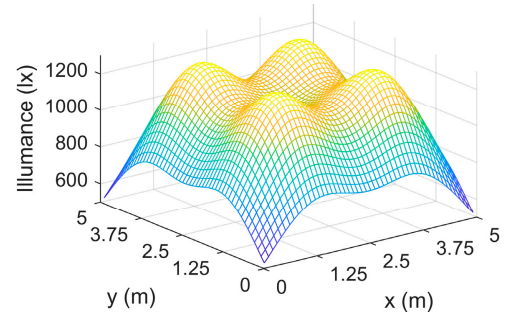

(a)

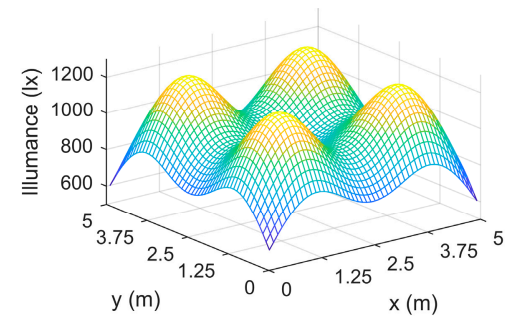

(b)

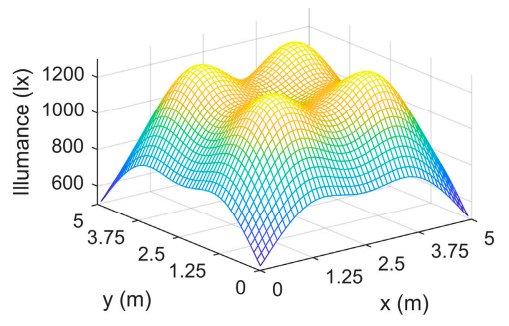

(c)

Figure 14. Illuminance distribution: (a) the illuminance distribution of the normal layout; (b) the illuminance distribution of the optimal layout of illumination; (c) the illuminance distribution of the optimal layout of channel quality.

Table 5. Illuminance data comparison.

\begin{tabular}{cccccc}
\hline Layout & $Q_{E}$ & $\begin{array}{c}\text { STD } \\
\mathbf{( 1 x )}\end{array}$ & $\begin{array}{c}\text { Min } \\
(\mathbf{l x})\end{array}$ & $\begin{array}{c}\text { Mean } \\
(\mathbf{l x})\end{array}$ & $\begin{array}{c}\text { Max } \\
(\mathbf{l x})\end{array}$ \\
\hline $\begin{array}{c}\text { Normal layout } \\
\text { Optimal layout }\end{array}$ & 6.2977 & 163.2313 & 524.9849 & 1027.9767 & 1281.1878 \\
$\begin{array}{c}\text { of illumination } \\
\begin{array}{c}\text { Optimal layout } \\
\text { of channel quality }\end{array}\end{array}$ & 6.0945 & 120.4515 & 606.2184 & 974.9943 & 1222.7475 \\
\hline
\end{tabular}

In Section 3, we found that the normal layout and the optimal layout of channel quality are so similar that the difference in the data of illuminance distribution of these layouts is too small. The standard deviation (STD) of the optimal layout of illumination is reduced by 42.7798 and 48.7914 to $120.4515 \mathrm{~lx}$ compared with the normal layout's 163.2313 lx and the optimal layout of channel quality's $169.2429 \mathrm{~lx}$.

In general, the illuminance distribution of the three layouts meets the standards of the International Organization for Standardization [8]. However, it is recommended to use the optimal layout of illumination in applications with high illumination uniformity requirements.

\subsection{SNR Distribution}

The SNR distribution of the three different LED layouts is simulated. The simulation results are shown in Figure 15. A received optical power of $\mathrm{SNR}=13.6 \mathrm{~dB}$ is required for a stable communication link [6]. In Figure 16, the dark area is the area in which SNR $<13.6 \mathrm{~dB}$. The data comparison is shown in Table 6.

From Table 6, the effective area percentage of the optimal layout of channel quality is increased by $0.32 \%$ and $6.08 \%$ to $88.80 \%$ as compared with the normal layout's $88.48 \%$ and the optimal layout of illumination's $82.72 \%$. The STD of SNR distribution of the optimal layout of channel quality is reduced by 0.0116 and 0.7338 to $0.7978 \mathrm{~dB}$ as compared with the normal layout's $0.8094 \mathrm{~dB}$ and the optimal layout of illumination's $1.5316 \mathrm{~dB}$. The minimum SNR distribution of the optimal layout of channel quality is increased by 0.1138 and 1.1945 to $13.0040 \mathrm{~dB}$ as compared with the normal layout's $12.8902 \mathrm{~dB}$ and the optimal layout of illumination's $11.8095 \mathrm{~dB}$. 


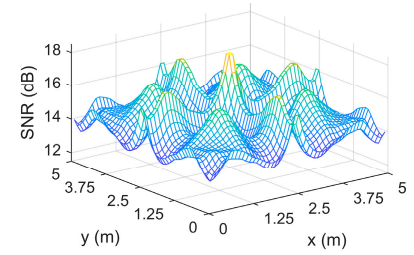

(a)

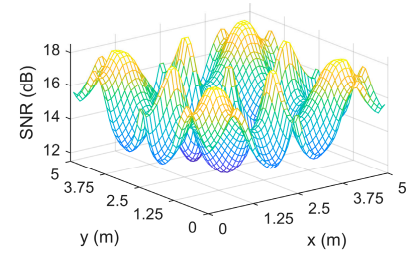

(b)

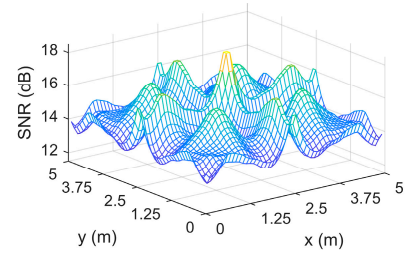

(c)

Figure 15. Signal-to-noise ratio (SNR) distribution: (a) the SNR distribution of the normal layout; (b) the SNR distribution of the optimal layout of illumination; (c) the SNR distribution of the optimal layout of channel quality.

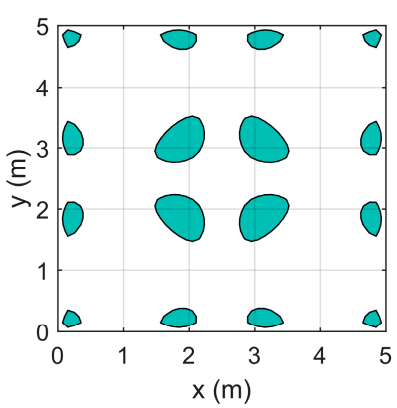

(a)

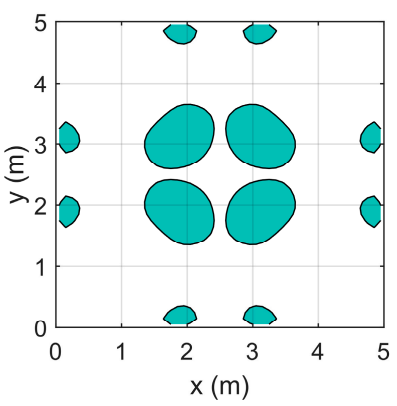

(b)

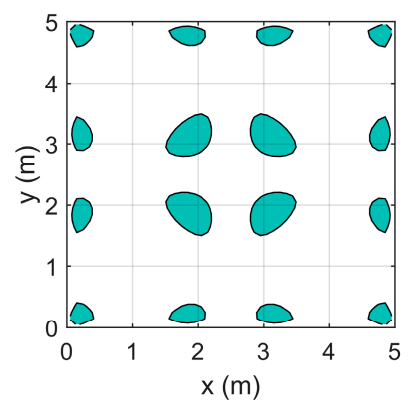

(c)

Figure 16. Noneffective area distribution: (a) the noneffective area distribution of the normal layout; (b) the noneffective area distribution of the optimal layout of illumination; (c) the noneffective area distribution of the optimal layout of channel quality.

Table 6. SNR data comparison.

\begin{tabular}{ccccccc}
\hline Layout & $Q_{S N R}$ & $\begin{array}{c}\text { STD } \\
(\mathbf{l x})\end{array}$ & $\begin{array}{c}\text { Min } \\
(\mathbf{l x})\end{array}$ & $\begin{array}{c}\text { Mean } \\
\mathbf{( l x )}\end{array}$ & $\begin{array}{c}\text { Max } \\
\mathbf{( l x})\end{array}$ & $\begin{array}{c}\text { Effective } \\
\text { Area } \\
\text { Percentage }\end{array}$ \\
\hline $\begin{array}{c}\text { Normal layout } \\
\begin{array}{c}\text { Optimal layout } \\
\text { of illumination }\end{array}\end{array}$ & 17.9596 & 0.8094 & 12.8902 & 14.5359 & 18.3151 & $88.48 \%$ \\
$\begin{array}{c}\text { Optimal layout } \\
\text { of channel quality }\end{array}$ & 18.1616 & 1.5316 & 11.8095 & 15.1084 & 18.2836 & $82.72 \%$ \\
\hline
\end{tabular}

In general, the normal layout and the optimal layout of channel quality are so similar that both of them can be chosen in general practical applications, but it is recommended to use the optimal layout of channel quality in applications with high SNR standards.

\subsection{M-DS Distribution}

In an indoor VLC system, there are lots of different channel paths; the arrival time of the signals via the paths differs due to the different path lengths, and the pulse width of the received signal is widened due to the multipath effect. The phenomenon is called delay spread.

Differently from most existing literature [1,2,5], in this paper, we consider the effect of FOV in M-DS distribution, so we get something new.

The M-DS refers to the difference in arrival time between the earliest arrival signal and the latest arrival signal. The M-DS distribution of the three different LED layouts is simulated. The simulation results are shown in Figure 17 and the data comparison is shown in Table 7. 


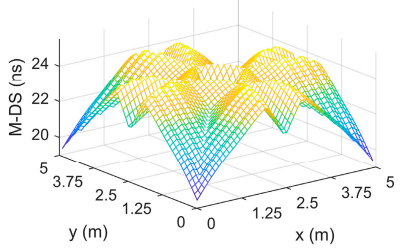

(a)

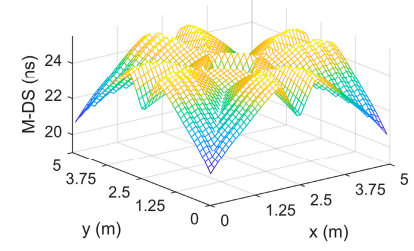

(b)

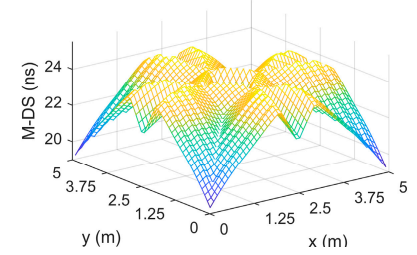

(c)

Figure 17. Maximum delay spread (M-DS) distribution: (a) the M-DS distribution of the normal layout; (b) the M-DS distribution of the optimal layout of illumination; (c) the M-DS distribution of the optimal layout of channel quality.

Table 7. M-DS data comparison.

\begin{tabular}{ccccc}
\hline Layout & $\begin{array}{c}\text { STD } \\
\text { (ns) }\end{array}$ & $\begin{array}{c}\text { Min } \\
\text { (ns) }\end{array}$ & $\begin{array}{c}\text { Mean } \\
\text { (ns) }\end{array}$ & $\begin{array}{c}\text { Max } \\
\text { (ns) }\end{array}$ \\
\hline $\begin{array}{l}\text { Normal layout } \\
\begin{array}{l}\text { Optimal layout } \\
\text { of illumination }\end{array}\end{array}$ & 0.9548 & 19.3973 & 22.9474 & 24.3332 \\
$\begin{array}{c}\text { Optimal layout } \\
\text { of channel quality }\end{array}$ & 0.8247 & 20.7084 & 23.7730 & 25.0527 \\
\hline
\end{tabular}

By comparing Figures 15 and 17, we find that, in general, where M-DS is smaller, SNR is larger. As for the difference in the data in the corner, it can be explained by the FOV. Due to the presence of the FOV, most signals with a long transmission path in the corner are not within its FOV and, therefore, not recorded, resulting in a large drop in the M-DS at that location. The same reason causes the ISI noise power and the signal power at the position to be correspondingly reduced, while the shot noise and thermal noise are constant. All these result in the M-DS in the corner being the shortest, but the SNR in the same position not being large.

From Table 7, the minimum M-DS distribution of the optimal layout of channel quality is reduced by 0.1093 and 1.4204 to $19.2880 \mathrm{~ns}$ as compared with the normal layout's $19.3973 \mathrm{~ns}$ and the optimal layout of illumination's $20.7084 \mathrm{~ns}$. The mean of the M-DS distribution of the optimal layout of channel quality is reduced by 0.0706 and 0.8962 to $22.8768 \mathrm{~ns}$ as compared with the normal layout's $22.9474 \mathrm{~ns}$ and the optimal layout of illumination's $23.7730 \mathrm{~ns}$. The maximum M-DS distribution of the optimal layout of channel quality is reduced by 0.0549 and 0.7744 to $24.2783 \mathrm{~ns}$ as compared with the normal layout's $24.3332 \mathrm{~ns}$ and the optimal layout of illumination's $25.0527 \mathrm{~ns}$.

\subsection{Impulse Response}

The impulse response of the three different LED layouts is simulated. The simulation results are shown in Figure 18.

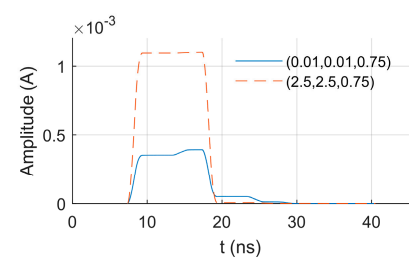

(a)

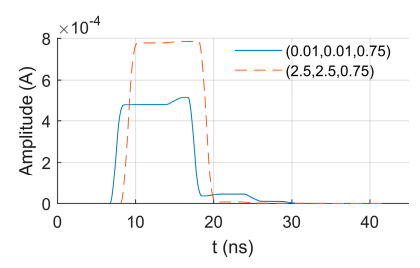

(b)

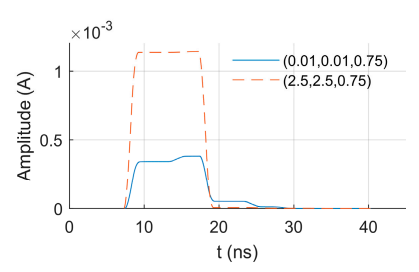

(c)

Figure 18. Impulse response: (a) the impulse response of the normal layout; (b) the impulse response of the optimal layout of illumination; (c) the impulse response of the optimal layout of channel quality.

Since the layouts of the normal layout and the optimal layout of channel quality are too close, the impulse responses of these are also similar. From Figure 18, there is no significant delay between the impulse response of the normal layout (optimal layout of channel quality) at the center and corner, and the 
difference between the amplitude of the two is large. There is a significant delay between the impulse response of the optimal layout of illumination at the center and corner, and the difference between the amplitudes of the two is smaller.

\section{Conclusions}

Considering the first reflected light, based on the conventional layout model and the classic indoor VLC model, we first defined the parameter $Q$ and then drew the corresponding curve of the layout and the parameter $Q$, thus determining the optimal layout of illumination and the optimal layout of channel quality. Combined with the normal layout, three layouts were determined. Later, an illuminance distribution simulation, SNR distribution simulation, M-DS distribution simulation, and IR simulation were performed for each layout. The achieved results show that the illuminance distributions of the three layouts meet the standards of the International Organization for Standardization. In the SNR distribution, M-DS distribution, and impulse response, the optimal layout of channel quality is obviously better than the optimal layout of illumination. In particular, the effective area percentage of the optimal layout of channel quality is increased by $0.32 \%$ and $6.08 \%$ to $88.80 \%$ as compared with the normal layout's $88.48 \%$ and the optimal layout of illumination's $82.72 \%$. However, compared with the normal layout, the advantages are not very prominent.

Author Contributions: Conceptualization, X.Z. and N.Z.; methodology, X.Z.; resources, X.Y.; data curation, X.Z.; writing—original draft preparation, X.Z.; writing_review and editing, M.B.K.; supervision, F.A.-T. and X.Y.; project administration, X.Y.; funding acquisition, X.Y. All authors have read and agreed to the published version of the manuscript.

Funding: This research was funded by National Natural Science Foundation of China, grant number 61301175.

Conflicts of Interest: The authors declare no conflict of interest.

\section{References}

1. Ding, J.; Huang, Z.; Ji, Y. Evolutionary Algorithm Based Power Coverage Optimization for Visible Light Communications. IEEE Commun. Lett. 2012, 16, 439-441. [CrossRef]

2. Wang, Z.; Yu, C.; Zhong, W.-D.; Chen, J.; Chen, W. Performance of a novel LED lamp arrangement to reduce SNR fluctuation for multi-user visible light communication systems. Opt. Express 2012, 20, 4564-4573. [CrossRef] [PubMed]

3. Borogovac, T.; Rahaim, M.; Carruthers, J.B. Spotlighting for visible light communications and illumination. In Proceedings of the 2010 IEEE Globecom Workshops, Miami, FL, USA, 6-10 December 2010; pp. 1077-1081. [CrossRef]

4. Wu, D.; Ghassemlooy, Z.; Leminh, H.; Rajbhandari, S.; Kavian, Y.S. Power distribution and Q-factor analysis of diffuse cellular indoor visible light communication systems. In Proceedings of the 16th European Conference on Networks and Optical Communications, Newcastle upon Tyne, UK, 20-22 July 2011; pp. 28-31.

5. Wu, D.; Ghassemlooy, Z.; Le Minh, H.; Rajbhandari, S.; Khalighi, M.A. Optimisation of Lambertian order for indoor non-directed optical wireless communication. In Proceedings of the 2012 1st IEEE International Conference on Communications in China Workshops (ICCC), Beijing, China, 15-17 August 2012; pp. 43-48.

6. Komine, T.; Nakagawa, M. Fundamental analysis for visible-light communication system using LED lights. IEEE Trans. Consum. Electron. 2004, 50, 100-107. [CrossRef]

7. Shen, Z.; Lan, T.; Wang, Y.; Wang, L.; Ni, G. Simulation and analysis for indoor visible-light communication based on LED. Infrared Laser Eng. 2015, 44, 2496-2500.

8. ISO. Lighting of Indoor Work Places; ISO 8995:2002(E)/CIE S 008/E-2001; ISO: Geneva, Switzerland, 2002.

Publisher's Note: MDPI stays neutral with regard to jurisdictional claims in published maps and institutional affiliations. 